\title{
Balancing Biases and Preserving Privacy on Balanced Faces in the Wild
}

\author{
Joseph P Robinson, Member, IEEE, Can Qin, Student Member, IEEE, Yann Henon, \\ Samson Timoner, Member, IEEE, and Yun Fu, Fellow, IEEE
}

\begin{abstract}
There are demographic biases in current models used for facial recognition (FR). Our Balanced Faces In the Wild (BFW) dataset serves as a proxy to measure bias across ethnicity and gender subgroups, allowing one to characterize FR performances per subgroup. We show performances are non-optimal when a single score threshold is used to determine whether sample pairs are genuine or imposter. Across subgroups, performance ratings vary from the reported across the entire dataset. Thus, claims of specific error rates only hold true for populations matching that of the validation data. We mitigate the imbalanced performances using a novel domain adaptation learning scheme on the facial features extracted using state-of-the-art. Not only does this technique balance performance, but it also boosts the overall performance. A benefit of the proposed is to preserve identity information in facial features while removing demographic knowledge in the lower dimensional features. The removal of demographic knowledge prevents future potential biases from being injected into decision-making. This removal satisfies privacy concerns. We explore why this works qualitatively; we also show quantitatively that subgroup classifiers can no longer learn from the features mapped by the proposed.
\end{abstract}

Index Terms_Facial recognition, Fair ML, Bias in Al, Balanced dataset, Imbalanced data problem, Domain adaptation.

\section{INTRODUCTION}

$\mathrm{T}$ HE more our society integrates with machine learning (ML), the higher the interest in topics such as bias, fairness, and the implications of using the technology [1]-[3]. And, the more we depend on technology that speeds up or automates everyday tasks, the more attention concepts such as biased and unfair algorithms should receive [4], [5]. Systems deployed for sensitive tasks, such as biometrics [6] (e.g., facial recognition (FR)), deserve thorough examinations. We aim to provide a test-bed for existing FR systems to report results fairly.

In FR, one typically trains a model on large-scale, paired face data and then deploys the model as a feature extractor. During enrollment (Fig. 1), features are stored to be later compared at inference time to the features of a test face. Today, models are convolutional neural networks $(\mathrm{CNNs})$ trained to represent face features in $\mathrm{N}$-dimensions with minimal distances between the same identity and maximum between unique identities.

Provided multiple face features encoded by a CNN, one learns a similarity score threshold to form a decision boundary separating the genuine scores from the imposter. This score threshold (i.e., $\theta$ ) determines whether to accept a pair as genuine. Face features are determined to originate from the same person if the score $s$ (or distance) satisfies a criterion from a single value [7]-[10]. Mathematically, a pair of faces match provided $s \geq \theta$. In the end, $\theta$ serves as a trade-off parameter concerning the false-positive (FP) and false-negative (FN) rates (Fig. 2).

In practice, specific sub-groups (e.g., ethnicity, gender, and other demographics) are generally not distributed in the held-out data set as they are during the operation of a system. The single global threshold can favor specific demographics. The optimal

- J.P. Robinson, C. Qin, and Y. Fu are with the College of Engineering, Northeastern University, Boston, MA, 02115.

- Y. Hann and S. Timoner are with ISM, Cambridge, MA, 02138.

Manuscript submitted 31 May 2021. threshold $\theta$ becomes a function of demographics (Fig 3). As shown, this problem is solvable via variable thresholds, i.e., sliding threshold set according to demographic information [11].

The adverse effects of a global threshold are three-fold: (1) Because the evaluation set is typically imbalanced, the reported performance ratings over-represent the demographic of some subgroups more than others. Hence, the algorithms favor the traits of the most common subgroups. (2) The score range between genuine pairs varies across demographics due to the CNN having different sensitivities to subgroup face features (Fig. 4). (3) The optimal score shifts across subgroups and, hence, a global threshold varies from the optimal.

To address the lack of balanced data (i.e., (1)), we built Balanced Faces In the Wild (BFW) to measure subgroup biases in FR (Fig. 5). BFW serves as a fair evaluation for FR systems by considering demographic-specific performances (Table 1). We use BFW to understand the performance gap (e.g., bias) present in facial features extracted from state-of-the-art (SOTA) CNNs. We then suggest a mechanism to rid the features of such bias and level-out performance ratings across demographics while also improving the overall accuracy. Specifically, we unlearn demographic knowledge in the features while preserving identity information. Thus, we learn to map the features to a lowerdimensional space containing less knowledge of subgroups. This feature adaptation scheme addresses items 2-3.

A byproduct of the proposed is relevant to problems in privacy. The learned features in the lower dimensional space contain less knowledge of the subgroups making it more difficult to extract the ethnicity and gender information from a face. Protecting user data is valuable as it lessens the chance that a malicious adversary will use it in detrimental ways [12]. It is therefore important for researchers to protect personal attributes of the user.

The contributions of this journal extension of [11] are:

- Demonstrate a bias in SOTA CNNs with our BFW dataset (Table 2). We propose a feature learning scheme that 


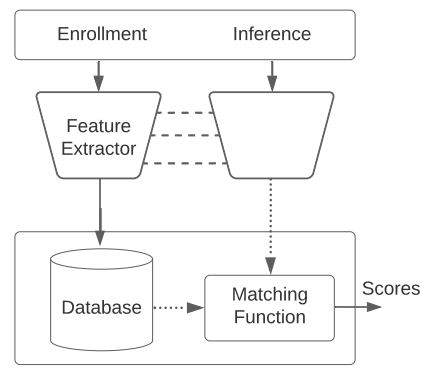

Fig. 1: Generic FR system. Enrollment: extract features from faces and store them in a database. Inference: extract features from a test face, and match to those in the database to produce scores.

de-biases face features and balances performances across subgroups, increasing overall performance.

- Minimize subgroup information in features - a byproduct of the proposed is the reduction of subgroup-based knowledge. This addresses privacy concerns, along with avoiding other potential biases, whether intentional (e.g., conscious decision making based on subgroups) or not (e.g., applied ML).

- Provide insights with analysis of challenging samples overcome by the proposed debiasing scheme.

We organized the rest of the paper as follows: in Section 2, we review related work. We go over the construction of the BFW database in Section 3. In Section 4, we introduce the proposed method. Section 5 follows the settings and results of the experiments. We conclude and discuss the next steps in Section 6.

\section{Related Work}

We discuss modern-day FR systems (i.e., SOTA neural network (NN)-based), along with the benchmark datasets and training procedures for these data-driven frameworks. We follow with a brief discussion on the supporting data focused on balance across label types. Then, we describe several related works in domain adaptation. Finally, we review the problems of privacy in FR.

\subsection{Bias in FR}

Modern deep models have worked with prominence in automatic face understanding. In 2014, Taigman et al. from Facebook first proposed using a deep CNN for FR [13]. Over a half of a decade later, there has been at least one significant contribution in conventional FR each year ever since (i.e., 2015 [14], 2016 [15], 2017 [8], 2018 [7], 2019 [16], and even 2020, the year of facial masks [17]). Scholars provide details on deep learning advances in FR technology as a part of recent surveys [18], [19], with another focused on bias specific to FR [20].

To address bias in FR, researchers focus on characterizing performance across soft attributes, such as gender [21], ethnicity, or age [6]. Researchers have spent significant efforts proposing problem statements and solutions to bias in FR tech [22], [23]. We focus on gender and ethnicity demographics (i.e., subgroups).

Balakrishnan et al. trained a generator to manipulate latent space features in that the controlled attributes were skin tone, length of hair, and hair color [24]. Another synthesis solution proposed was to generate faces across various ages to augment training data [25]. Around the same time, Muthukumar et al. studied the effects color tone has on gender classification by

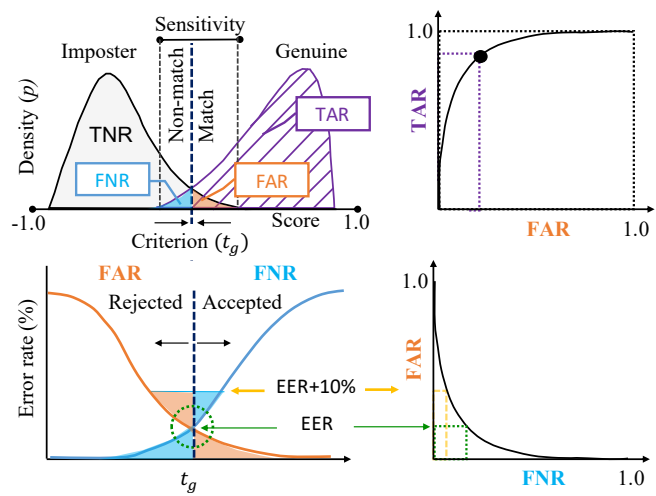

Fig. 2: Depiction of biometrics. The signal detection model (SDM) shows the sensitivity of a single threshold $t_{g}$ (top-left). The area to the right of the threshold considers all accepted pairs, both correctly and incorrectly predicted. True Acceptance Rate (TAR) as a function of FAR is a common way to report ratings for given false rates (topright). Equally common in FR is the trade-off between FNR and FAR (bottom-left and bottom-right).

recoloring faces in images across a spectrum of brightness that spans from a lighter to a darker tone while holding all other features constant [26]. Terhorst et al. recognized the same phenomena we found in our work: the variation in the sensitivities of scores for different demographics [27]. Specifically, the authors propose a score normalization scheme to handle the problem of inaccurate performance ratings when comparing demographicspecific performances to the average-a problem we highlighted in [11], and which we now extend.

Some aim to characterize the amount of bias in a system, whether for gender [28]-[30], ethnicity, age [31], or more than one [32]-[36]. A recent European Conference on Computer Vision (ECCV) challenge provided an incentive for researchers to tackle bias concerning ethnicity, gender, age, pose, and with and without sunglasses [37]. Other recent works in face recognition (FR) technology introduce additional modalities, such as profile information, to the problem of bias [38], [39]. Other questions concern the measures of biases in FR systems [28], [33], FR templates [40], score level biases [27], and biases in existing (i.e., trained) models [41]. Wang et al. introduced a reinforcement learning-based race balanced network to find optimal margins for non-Caucasians as a Markov decision process before passing to the deep model that learns policies for an agent to select margins as an approximation of the Q-value function- it reduces variations in scattering between features across races [42]. There has even been the introduction of HCI-based views as semisupervised bias detection systems that act as tools with humans in the loop [43]. The aforementioned differs from the proposed by working in image-space, whereas we target the features extracted from existing models, and without retraining the original model.

\subsection{Imbalanced data and data problems in FR}

The impact of the quality of fairness depends on the context. There have been many proposals of various paradigms to a solution: some alter the data distribution to yield classifiers of equal performances for all classes (i.e., re-sampling, like by under-sampling and over-sampling [44]); others alter the data itself (i.e., algorithms that adjust classification costs). For instance, Oquab et al. re-sampled at the image patch-level [45]. 


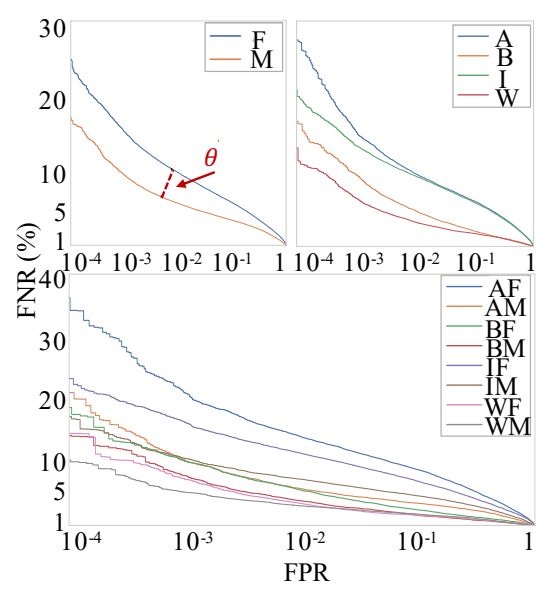

Fig. 3: Detection error trade-off (DET) curves. Top-left: per gender. Top-right: per ethnicity. Bottom: per subgroup (i.e., combined). Dashed line shows about $2 \times$ difference in FPR for the same threshold $\theta$. FNR is the match error count (closer to the bottom is better).

Specifically, the aim was to balance foregrounds and backgrounds for object recognition. Rudd et al. proposed the mixed objective optimization network (MOON) architecture [46] that learns to classify attributes of faces by treating the problem as a multi-task (i.e., a task per attribute) attribute to more balanced performances when training on data that has an imbalanced distribution across attributes. The Cluster-based Large Margin Local Embedding (CLMLE) [47] sampled clusters of samples in the featurespace regularized the models at the decision boundaries of underrepresented classes. Wang et al. modified images by masking out aspects of humans that cause "leakage" of gender information, as a means to avoid biasing a set of labels describing a scene to that of a specific gender [48]. See literature reviews on approaches that solutions at the data or algorithmic level [49]-[51].

More specific to faces, Drozdowski et al. summarized that the cohorts of concern in biometrics are demographic (e.g., sex, age, and race), person-specific (e.g., pose or expression [52], and accessories like eye-wear or makeup), and environmental (e.g., camera-model, sensor size, illumination, and occlusion) [6]. Albiero et al. found empirical support that having training data that is well balanced in gender does not mean that results of a gender-balanced test set will be fair [53]. We study the effect demographics have in facial verification (FV) by assessing demographic-specific performances. Our BFW data resource allows us to analyze existing SOTA deep CNNs on different demographics (or subgroups). We provide practical insights showing that experiments often report misleading performance ratings that depend on the population's demographics.

To match the capacity of modern-day deep models, many released large FR datasets [54]-[57]. More recently, several focused on balancing the demographics in FR data [3], [58][60]. Diversity in Faces $(\mathrm{D} i \mathrm{~F})$ came first [60], which did not include identity labels. $\mathrm{DiF}$ is no longer available for download. Others released data with demographics balanced and did not include identity labels [3], [59]. Hupont et al. proposed DemogPairs balanced across six subgroups of 600 identities from CASIA-WebFace (CASIA-W) [61], VGG [55], and VGG2 [56]. Our BFW includes eight subgroups (i.e., split the African/Indian subgroup used in DemogPair into separate groups, Black and

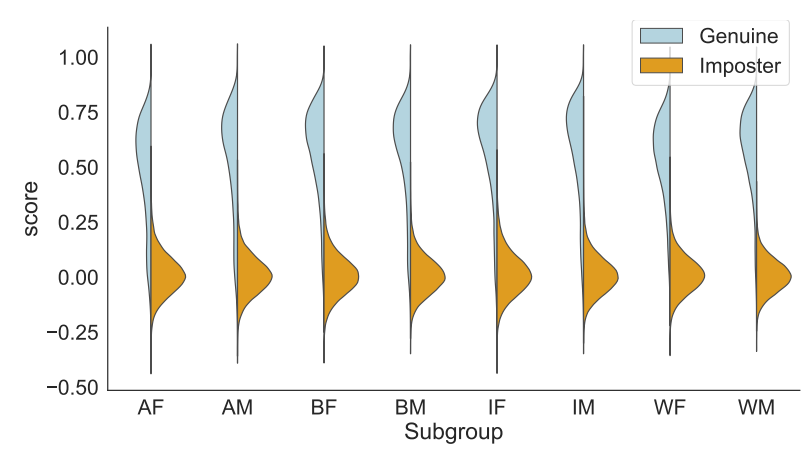

Fig. 4: SDM across subgroups. Scores of imposters have medians $\approx 0.0$ but with variations in upper percentiles; genuine pairs vary in mean and spread (e.g., AF has more area of overlap). A threshold varying across different subgroups yields a constant FAR.

Indian), 800 identities, and more face samples per identity. Additionally, we only used the VGG datasets and not CASIAWeb to test a broader range of models (i.e., even models trained on CASIA-Web). Recognizing that someone already trained existing SOTA models on a public resource, we built BFW from just VGG2 to minimize conflicts in the overlap between train and test. Table 1 compares our data with the others.

\subsection{Feature Alignment / Domain Adaptation}

Domain adaptation (DA) [62]-[64] employs labeled data from the source domain to make it generalize well to the typically label-scarce target domain; hence, a standard solution to relieve annotation costs. We can roughly classify DA as the semisupervised DA [64] or the unsupervised one [65] according to the access to target labels. The crucial challenge toward DA is the distribution shift of features across domains (i.e., domain gap), which violates the distribution-sharing assumption of conventional machine learning problems. In our case, the different domains are the different subgroups (Table 2).

Some Feature Alignment (FA) methods attempt to project the raw data into a shared subspace where certain feature divergence or distance confuses groups. Many developed methods in this line, such as Correlation Alignment (CORAL) [66], Maximum Mean Discrepancy (MMD) [67], and Geodesic Flow Kernel (GFK) [68], [69]. Currently, adversarial domain alignment methods (i.e., DANN [70], ADDA [71]) have attracted increasing attention by designing a zero-sum game between a domain classifier (i.e., discriminator) and a feature generator. The discriminator could not differentiate the source and target features if it mixed the features of different domains up. More recently, learning well-clustered target features proved helpful in conditional distribution alignment. Both DIRT-T [65] and MME [64] methods applied an entropy loss on target features to group them implicitly as multiple clusters in the feature space to keep the discriminative structures through adaptation. Inspired by FA, we aim to align the score distributions of the different subgroups by adjusting the sensitivities in true scores (Fig. 4).

\subsection{Protecting demographic information in FR}

Several recent attempts to solving bias in subgroup-based classifiers (e.g., ethnicity and gender classifiers): attempts to disguise demographic information in faces while preserving identities for privacy and protection purposes [72]-[74]. 
TABLE 1: BFW features compared to related resources. Note the balance across identity (ID), gender (G), and ethnicity (E). Compared with DemogPairs, BFW provides more samples per subject and subgroups per set. Also, BFW uses a single resource, VGG2. All the while, RFW supports a different task (i.e., subgroup classification). RFW and FairFace focus on race distribution without the support of identity labels. Code available to explore BFW and reproduce its experiments (https://github.com/visionjo/facerec-bias-bfw).

\begin{tabular}{|c|c|c|c|c|c|c|c|}
\hline \multicolumn{2}{|c|}{ Database } & \multicolumn{3}{|c|}{ Number of } & \multicolumn{3}{|c|}{ Balanced Labels } \\
\hline Name & Source & Faces & IDs & Subgroups & ID & $\mathrm{E}$ & $\mathrm{G}$ \\
\hline RFW [3] & MS-Celeb-1M & $\approx 80,000$ & $\approx 12,000$ & 4 & $x$ & $\checkmark$ & $x$ \\
\hline DemogPairs [58] & CASIA-W, VGG $(+2)$ & 10,800 & 600 & 6 & $\checkmark$ & $\checkmark$ & $\checkmark$ \\
\hline FairFace [59] & Flickr, Twitter, Web & 108,000 & - & 10 & $x$ & $\checkmark$ & $\checkmark$ \\
\hline BFW (ours) [11] & VGG2 & 20,000 & 800 & 8 & $\checkmark$ & $\checkmark$ & $\checkmark$ \\
\hline
\end{tabular}

TABLE 2: Data statistics, nomenclature, and scores for subgroups as part of our BFW data. Top: Specifications of BFW and subgroup definitions. Middle: Number of pairs for each partition. Bottom: Accuracy using a global threshold $t_{g}$, the value of the optimal threshold $t_{o}$, and accuracy using $t_{o}$ (bottom) per subgroup. We group columns by race and then further split by gender. Notice that ratings are inconsistent across subgroups. The acronyms for the subgroups are used throughout the manuscript.

\begin{tabular}{|c|c|c|c|c|c|c|c|c|c|}
\hline & \multicolumn{2}{|c|}{ Asian (A) } & \multicolumn{2}{|c|}{ Black (B) } & \multicolumn{2}{|c|}{ Indian (I) } & \multicolumn{2}{|c|}{ White (W) } & \multirow[b]{2}{*}{ Aggregated } \\
\hline & Female (AF) & Male (AM) & $\mathrm{BF}$ & $\mathrm{BM}$ & IF & $\mathrm{IM}$ & WF & WM & \\
\hline No. Faces & 2,500 & 2,500 & 2,500 & 2,500 & 2,500 & 2,500 & 2,500 & 2,500 & 20,000 \\
\hline No. Subjects & 100 & 100 & 100 & 100 & 100 & 100 & 100 & 100 & 800 \\
\hline No. Faces / subject & 25 & 25 & 25 & 25 & 25 & 25 & 25 & 25 & 25 \\
\hline No. Positive & 30,000 & 30,000 & 30,000 & 30,000 & 30,000 & 30,000 & 30,000 & 30,000 & 240,000 \\
\hline No. Negative & 85,135 & 85,232 & 85,016 & 85,141 & 85,287 & 85,152 & 85,223 & 85,193 & 681,379 \\
\hline Total & 115,135 & 115,232 & 115,016 & 115,141 & 115,287 & 115,152 & 115,223 & 115,193 & 921,379 \\
\hline Acc@ $t_{g}$ & 0.876 & 0.944 & 0.934 & 0.942 & 0.922 & 0.949 & 0.916 & 0.918 & $0.925 \pm 0.022$ \\
\hline$t_{o}$ & 0.235 & 0.274 & 0.267 & 0.254 & 0.299 & 0.295 & 0.242 & 0.222 & $0.261 \pm 0.025$ \\
\hline$A c c @ t_{o}$ & 0.916 & 0.964 & 0.955 & 0.971 & 0.933 & 0.958 & 0.969 & 0.973 & $0.955 \pm 0.018$ \\
\hline
\end{tabular}

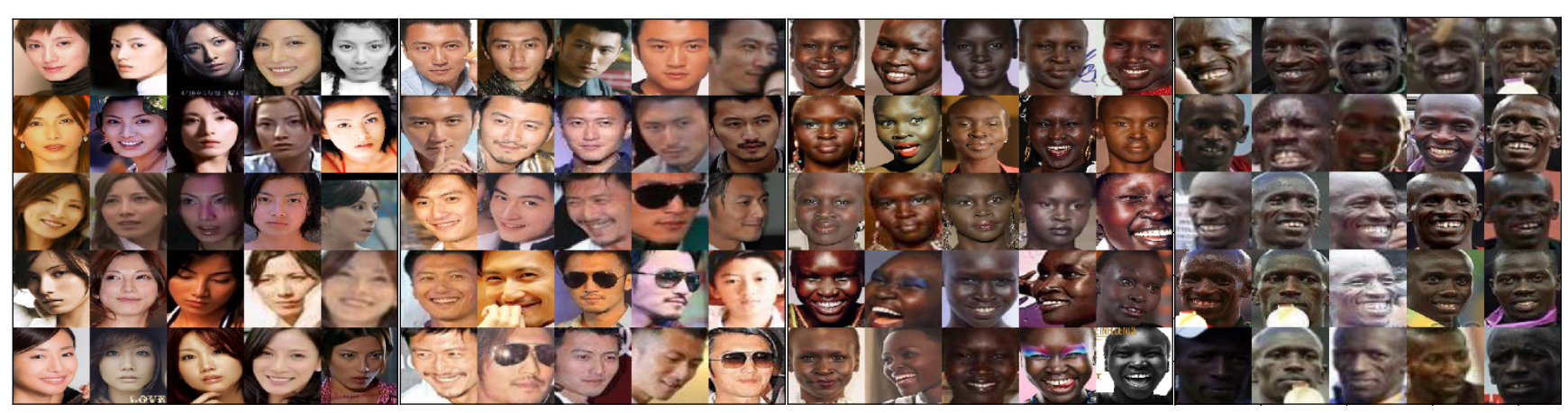

(a) Asian-Female (AF).

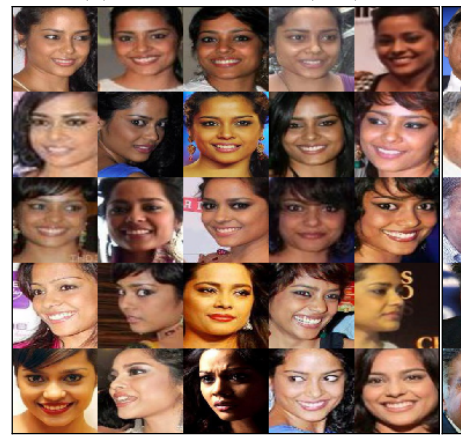

(e) Indian-Female (IF). (b) Asian-Male (AM).

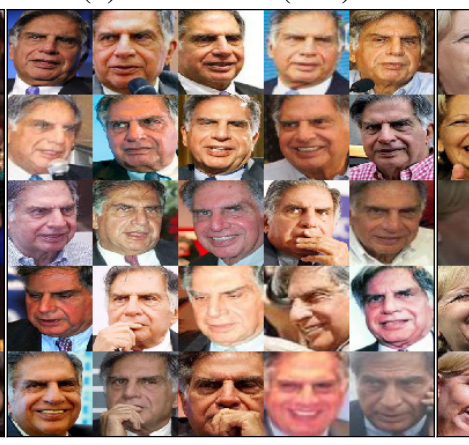

(f) Indian-Male (IM). (c) Black-Female $(\mathrm{BF})$.

(d) Black-Male (BM).

Fig. 5: Samples of BFW. For each subgroup, we show 25 samples for a single, randomly selected subject. 
Prior works recognized the importance of preserving the identity information in facial features while ridding it of evidence of demographics. Our model inherently does this as part of the target, aiming for an inability to recognize subgroups. Some achieved this using adversarial learning on top of the features via a Minimax filter [75]: maximizing the attributes loss while minimizing the target task. Later on, several approached it as a minimization problem by reversing the gradient of the protected classifier. For instance, Bertran et al. learned a projection that maps images to an embedding space such that gender information cannot be inferred [76]. Similar to this, we also aim to rid the data of demographic knowledge; however, the difference is that we learn to protect demographics in the facial features often stored in place of imagery (i.e., we learn to map from a bias to a non-bias feature space). Ray et al. followed the same path (i.e., mapping images to space with demographic information protected) [77]. Again, we aim to protect a database of facial features, with no assumption that the initial model or the raw images are accessible. Besides, Wu et al. learned to rid knowledge of gender in features from videos with people performing actions [78], [79]. For this, features were extracted from video data to classify actions using a representation containing no knowledge of the demographics of the subject performing. Hence, we focus on an entirely different problem, that of fine-grained FR.

Additionally, Wu et al. focused on concepts of ridding identity information as seen by a task-specific ensemble of models by modifying video data in image-space [78]. For instance, by cropping out faces or the entire bodies of subjects in the video. We use a single model to modify the facial features by learning a mapping to a feature space where the protected variables are unrecoverable but without compromising the performance of the FR system, which we improve. Existing works are broadly similar for being motivated by privacy concerns but differ in the ideology, methodology, and even the problem statements, data, and tasks.

Those above assume the facial encoding is accessible - this makes sense in terms of reduced computations (i.e., no need to encode each time) and storage (i.e., features are smaller representations of the image). However, several works aimed to hide attribute information in image space; for instance, Othman et al. learned to morph faces to suppress gender information in the image space while preserving the identification [81]. Guo et al. proposed a mapping from image to noise, encrypting the image such that the encoder still decodes the identity but without the ability to determine gender by machine or human [82]. Ma et al. viewed the communication between serves as the point of privacy concern-a lightweight privacy-preserving adaptive boosting (AdaBoost) FR framework (POR) based on additive fusion for secret sharing to encrypt model parameters while using a cascade of classifiers to address different protocols [83].

\section{BaLanced Faces In the WiLd (BFW)}

BFW provides balanced data across ethnicity (i.e., Asian (A), Black (B), Indian (I), and White (W)) and gender (i.e., Female (F) and Male (M))-eight demographics referred to as subgroups (Fig. 5). As listed in Table 1, BFW has an equal number of subjects per subgroup (i.e., 100 subjects per subgroup) and faces per subject (i.e., 25 faces per subject). Note that the key difference between BFW and DemogPairs is in the additional attributes and the increase in overall labeled data; the differences between RFW and FairFace are in the identity labels and distributions (Table 2).
We built BFW with VGG2 [56] by using classifiers on the list of names and then the corresponding face data. Specifically, we ran a name-ethnicity classifier [84] to generate the initial list of subject proposals. Then, the corresponding faces with ethnicity [85] and gender [86] classifiers further refined the list. Next, we manually validated, keeping only those that were genuine members of the respective subgroup. We then limited faces for each subject to 25 faces selected at random. Thus, BFW costs minimal human input, having had the proposal lists generated by automatic machinery.

In summary, a total of four experts in FR had manually validated all of the data: first, the validation of individuals per subgroup was conducted (i.e., inspect that all subjects belong to the assigned subgroups), and then faces of the individual (i.e., verify that each face instance belongs to the identity). In both cases, only the subjects and samples per subgroup and identity, respectively, that agreed upon by all four annotators were kept. We refer the reader to our conference paper for more details [11].

We determined the subgroups of BFW based on physical features most common amongst the respective subgroup [11]. We can regard this as multiple domains because of the feature distribution mismatch across these subgroups. However, the assumption that a discrete label can describe an individual is imprecise. The assumption allows for a finer-grain analysis of the subgroup and is a step in the right direction. Thus, we refute any claim that our efforts here are the ultimate solution; the data and proposed machinery are merely an attempt to establish a foundation for future work to extend. The two genders for the four ethnic groups make up the eight subgroups of the BFW dataset (Fig. 5). Formally put, the tasks addressed have labels for gender $l^{g} \in\{F, M\}$ and ethnicity $l^{e} \in\{A, B, I, W\}$, where the $K$ subgroups (i.e., demographics) are then $K=\left|l_{g}\right| *\left|l_{e}\right|=8$.

\section{Methodology}

To discuss the bias and privacy concerns addressed by the proposed, we first introduce facial verification (FV). Specifically, we review the problem statement, the supporting facial image dataset, along with the proposed framework set to achieve the solutions sought in this work. To preserve identity information while balancing the sensitivities of features for the different demographics (i.e., subgroups), and to remove knowledge of the subgroups from the facial encoding - the typical representation available for operational cases.

\subsection{Problem statement}

FV systems decide based on the likeliness a pair of faces are of the same identity. In fact, the core procedure of verification systems is often similar to the FR employed for various applications. Specifically, we train a Convolutional Neural Network (CNN) on a closed set of identities, and then later used it to encode faces (i.e., map face images to features). The features are then compared in closeness to produce a single score-often via cosine similarity in FR [87]. It is imperative to learn the optimal score threshold separating true from false pairs. The threshold is the decision boundary in score space, i.e., the matching function.

\subsubsection{The matching function}

A real-valued similarity score $\mathrm{R}$ assumes a discrete label of $Y=1$ for genuine pairs (i.e., a true-match) or $Y=0$ for imposter (i.e., untrue-match). We map the actual real number to a discrete label 


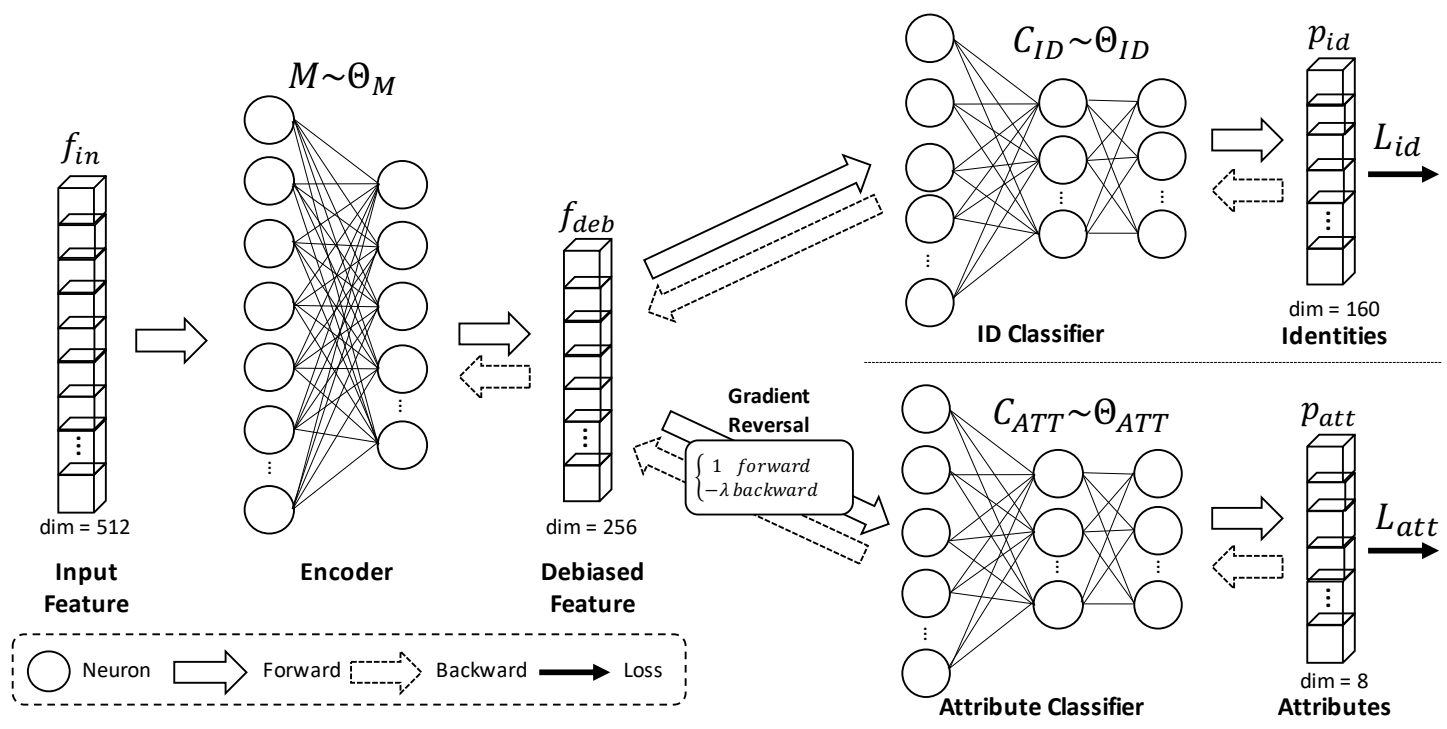

Fig. 6: Debiasing framework. The framework used to learn a projection that casts facial features to a space that (1) preserves identity information (i.e., $C_{I D}$ ) and (2) removes the knowledge of subgroup (i.e., $C_{A T T}$ ). The benefits of this are two-fold: the ability to verify pairs of faces fairly across attributes and an inability to classify attributes for privacy and safety purposes. Note that the gradient reversal [80] flips the sign of the error back-propagated from $C_{A T T}$ to $M$ by scalar $\lambda$ during training.

by $\hat{Y}=\mathbb{I}\{\mathrm{R}>\theta\}$ for some pre-defined threshold $\theta$. We can express the aforementioned as matcher $d$ operating as

$$
f_{\text {boolean }}\left(\vec{x}_{i}, \vec{x}_{j}\right)=d\left(\vec{x}_{i}, \vec{x}_{j}\right) \leq \theta,
$$

where the face features in $\vec{x}$ being the $i^{t h}$ and $j^{t h}$ sample- a conventional scheme in the FR research communities [88]. We use cosine similarity as the matcher in Eq. 1, which produces a score in closeness for the $i^{\text {th }}$ and $j^{\text {th }}$ faces (i.e., $l^{\text {th }}$ face pair) by $d\left(\vec{x}_{i}, \vec{x}_{j}\right)=s_{l}=\frac{f_{i} \cdot f_{j}}{\left\|f_{i}\right\|_{2}\left\|f_{j}\right\|_{2}}$. The decision boundary formed by threshold $\theta$ controls the level of acceptance and rejection. Thus, $\theta$ inherits a trade-off between sensitivity and specificity. The operating point chosen, almost always, depends on the purpose of the respective system (i.e., perhaps higher sensitivity (or lower threshold) for threat-related tasks, in that, flagging a few extra is worth not overlooking the one true positive). Specifically, the trade-off involves FNR that attempts to pass but falsely rejects-a Type 1 Error. Mathematically, it relates by

$$
\mathrm{FNR}=\frac{\mathrm{FN}}{\mathrm{P}}=\frac{\mathrm{FN}}{\mathrm{FN}+\mathrm{TP}}=1-\mathrm{TAR}=1-\frac{\mathrm{TP}}{\mathrm{FN}+\mathrm{TP}},
$$

with positive counts $\mathrm{P}$, true-positive (TP), and false-negative (FN).

The other error type contributes to the FPR, the Type II Error, which is when an imposter falsely passes:

$$
\mathrm{FAR}=\frac{\mathrm{FP}}{\mathrm{N}}=\frac{\mathrm{FP}}{\mathrm{FP}+\mathrm{TN}}=1-\mathrm{TNR}=1-\frac{\mathrm{TN}}{\mathrm{FP}+\mathrm{TN}},
$$

where the number of negatives is $N$, and the metrics are truenegative (TN), false-positive (FP), true-negative rate (TNR), and FAR. A matching function is a module typically characterized using the listed metrics (Fig 1). The geometric relationships of the metrics related to the score distributions and the choice of threshold show the trade-offs in error rates (Fig. 2).

The hyper-parameter $\theta$ determines the desired error rate on held-out validation, which is specific to the use-case. Researchers tend to set it for top performance. Some analyze $\theta$ as a range of values, for a more complete characterization is often obtainable with evaluation curves that inherently show performance tradeoffs. However, the held-out validation and test sets typically share data distributions as a single source partitioned into subsets (i.e., train, validation, test). Regardless, we transfer the decision boundary in score space that maximizes the performance to the pin-point (i.e., 1D) decision boundary. In our case, using cosine similarity, the value is a floating-point value spanning $[0,1]$.

\subsubsection{Feature alignment}

The tuple $\mathcal{D}=\left\{\left(\mathbf{x}_{i}, y_{i}\right) \in \mathcal{X} \times \mathcal{Y}\right\}_{i=1}^{N}$ represents domain $\mathcal{D}$, with $\mathcal{X}$ and $\mathcal{Y}$ representing the input feature space and output label space, respectively. The aim of FR algorithms is to learn a mapping function (i.e., a hypothesis): $\eta: \mathcal{X} \rightarrow \mathcal{Y}$, which assigns each sample vector with a semantic identity label.

In DA, we train source data and deploy on target data, where an abundance of paired data is available to train a model for a task similar to the target. Mathematically, we denote the labeled source domain $\mathcal{D}_{S}$ and the unlabeled target domain $\mathcal{D}_{T}$ as $\mathcal{D}_{S}=$ $\left\{\left(\mathbf{x}_{i}^{s}, y_{i}^{s}\right) \in \mathcal{X}_{S} \times \mathcal{Y}_{S}\right\}_{i=1}^{N_{S}}$ and $\mathcal{D}_{T}=\left\{\mathbf{x}_{i}^{t} \in \mathcal{X}_{T}\right\}_{i=1}^{N_{T}}$ with the sample count $N_{S}=\left|\mathcal{D}_{S}\right|$ and $N_{T}=\left|\mathcal{D}_{T}\right|$ corresponding to the $i$-th sample (i.e., $\mathbf{x}_{i} \in \mathbb{R}^{d}$ ) and label (i.e., $y_{i} \in\{1, \ldots, K\}$ ). $\mathcal{D}_{S}$ and $\mathcal{D}_{T}$ are further defined by tasks $\mathcal{T}_{S}$ and $\mathcal{T}_{T}$, which show the exact label type(s) and the specific $K$ classes of interest. The goal is to learn an objective $\eta_{S}: \mathcal{X}_{S} \rightarrow \mathcal{Y}_{S}$, and then transfer to target $\mathcal{D}_{T}$ for $\mathcal{T}_{T}$. By this, we leverage knowledg from both $\mathcal{D}_{S}$ for $\mathcal{D}_{T}$ to get $\eta_{T}$. Since either domain has different marginal distributions (i.e., $p\left(\mathbf{x}^{s}\right) \neq p\left(\mathbf{x}^{t}\right)$ ) and distinct conditional distributions (i.e., $\left.p\left(y^{t} \mid \mathbf{x}^{s}\right) \neq p\left(y^{t} \mid \mathbf{x}^{t}\right)\right)$ a model trained on the labeled source usually performs poorly on the unlabeled target. A typical solution towards such a domain gap is to learn a model $f$ that aligns the features in a shared subspace by $p\left(f\left(\mathbf{x}^{s}\right)\right) \approx p\left(f\left(\mathbf{x}^{t}\right)\right)$.

\subsection{Proposed framework}

Both identity and subgroup labels are used for the two objectives of the proposed framework (Fig. 6). Specifically, $\mathcal{D}=$ $\left\{\mathbf{x}_{i}, y_{i}^{i d}, y_{i}^{a t t}\right\}_{i=1}^{N}$, where $\mathbf{x} \in \mathbb{R}^{d}, y^{i d} \in\{1, \ldots, I\}$ and 


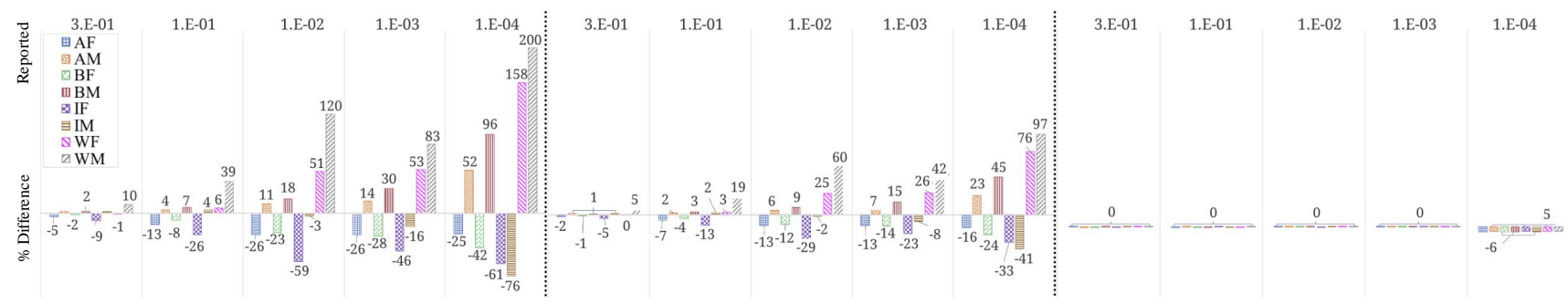

Fig. 7: The percent differences from the intended FPR across subgroups. Left: global threshold $\left(t_{g}\right)$ yields an FPR that spans up to $200 \%$ of the intended (i.e., WM for 1e-4); the F subgroups tend to perform worse than intended for all, while M's overshoot the intended performances besides IM at FPR=1e-4. Right: Subgroup-specific (or optimal) thresholds $t_{o}$ reduces the difference closer to zero. Furthermore, the proposed method (middle), which does not assume knowledge of attribute information at inference like for $t_{o}$, clearly mitigates the issue of the inconsistencies in the true versus reported FPR. Similar to the results in Table 3, the variations are nearly halved: the percent difference for subgroups is more balanced using the features adapted with our approach versus the baseline.

$y^{\text {att }} \in\{1, \ldots, K\}$. Hence, we aim to learn a mapping $\mathbf{f}_{d e b}=$ $M\left(\mathbf{x}, \Theta_{M}\right)$ to a lower-dimensional space $\mathbf{f}_{d e b} \in \mathbb{R}^{d / 2}$ that preserves the identity information of the target. We call this the identity loss $\mathcal{L}_{I D}$. We also learn to do so without subgroup information, which we call the attribute (or subgroup) loss $\mathcal{L}_{A T T}$. We form the total loss (i.e., the final objective $\mathcal{L}=\mathcal{L}_{I D}+\mathcal{L}_{A T T}$ ) by summing the two aforementioned loss functions:

$$
\begin{aligned}
\mathcal{L}_{I D} & =-\frac{1}{N} \sum_{i=1}^{N} \sum_{k=1}^{I} \mathbf{1}_{\left[k=y_{i}^{i d}\right]} \log \left(p\left(y=y_{i}^{i d} \mid \mathbf{x}_{i}\right)\right), \\
\mathcal{L}_{A T T} & =-\frac{1}{N} \sum_{i=1}^{N} \sum_{k=1}^{K} \mathbf{1}_{\left[k=y_{i}^{a t t}\right]} \log \left(p\left(y=y_{i}^{a t t} \mid \mathbf{x}_{i}\right),\right.
\end{aligned}
$$

where $p\left(y=y_{i}^{i d} \mid \mathbf{x}_{i}\right)$ and $p\left(y=y_{i}^{a t t} \mid \mathbf{x}_{i}\right)$ represent the softmax conditional probability of its identity and attribute.

We added $\mathcal{L}_{A T T}$ to debias the features to remove variation in scores that were previously handled with a variable threshold. Further, a byproduct is these features that preserve identity information without containing knowledge of subgroup- a critical concern in the privacy and protection of biometric data.

There are three groups of parameters (i.e., $\Theta_{M}, \Theta_{I D}$, and $\Theta_{A T T}$ ) required to be optimized by the objective (Fig. 6). Both the identity classifier $C_{I D}$ and attribute classifier $C_{A T T}$ are used to find a feature space that remains accurate to identity and not for subgroup by minimizing the empirical risk of $\mathcal{L}_{I D}$ and $\mathcal{L}_{A T T}$ :

$$
\begin{gathered}
\Theta_{I D}^{*}=\underset{\Theta_{I D}}{\arg \min } \mathcal{L}_{I D}, \\
\Theta_{A T T}^{*}=\underset{\Theta_{A T T}}{\arg \min } \mathcal{L}_{A T T} .
\end{gathered}
$$

It is important to note that the task of $\mathcal{L}_{A T T}$ is to be incorrect (i.e., learn a mapping that contains no knowledge of subgroup). Thus, a gradient reversal layer [80] that acts as the identity during the forward pass, while inverting the sign of the gradient backpropagated with a scalar $\lambda$ as the adversarial loss during training:

$$
\Theta_{M}^{*}=\underset{\Theta_{M}}{\arg \min }-\lambda \mathcal{L}_{A T T}+\mathcal{L}_{I D}
$$

Although the proposed learning scheme is simple, it proved effective for both objectives we seek to solve. The effectiveness is clearly demonstrated in the results and analysis.

\section{EXPERIMENTS}

We include two sets of experiments to show the effectiveness of the proposed using our balanced BFW [11]. First, we evaluate verification performance. Specifically, we compare the reported results compared to the actual results per subgroup. Then, for the privacy-preserving claim, we compare the performance of models trained on top of debiased features $f_{d e b}$ with those of the original features $f_{i n}$. For each, we present the problem statement, metrics and settings, and analysis. Finally, we do an ablation study to show the performance of the renowned LFW benchmark [88].

\subsection{Common settings}

We encode the baseline (i.e., $f_{\text {in }}$ ) using Arcface [9] (i.e., ResNet34). ${ }^{1}$ MS1M [54] was the train set, providing about 5.8M faces for $85 \mathrm{k}$ subjects. We prepared the faces using MTCNN [89] to detect five facial landmarks. We then applied a similarity transformation to align the face by the five detected landmarks, from which we cropped and resized each to $96 \times 112$. The RGB (i.e., pixel values of $[0,255]$ ) were normalized by centering about 0 (i.e., subtracting 127.5) and then standardizing (i.e., dividing by 128); features were later L2 normalized [90]. The batch size was 200, and a stochastic gradient descent optimizer with a momentum 0.9 , weight decay $5 \mathrm{e}-4$, and learning rate started at 0.1 and decreased by a factor of 10 two times when the error leveled. The choice in these settings was made based on Arcface being among the best performing FR deep models to date, and as it has become a popular choice for an off-the-shelf option for face recognition technology and applications.

For all experiments we used our BFW dataset (Section 3): the debias and privacy-based experiments use the pre-defined fivefolds $^{2}$; the ablation study on LFW uses all of BFW to train $\mathrm{M}$ (Fig. 6). As mentioned, we built BFW using data of VGG2, and there is no overlap with CASIA-Webface and LFW used to train the face encoder and for the ablation, respectfully.

\subsection{Debias experiment}

Typical FR systems are graded by the percent error - whether to a customer, prospective staff, and so forth. In other words, specialized curves, confusion matrices, and other metrics are not always the best way to communicate system performances to nontechnical audiences. It is better to discuss ratings in a manner

1. https://github.com/deepinsight/insightface

2. https://github.com/visionjo/facerec-bias-bfw 


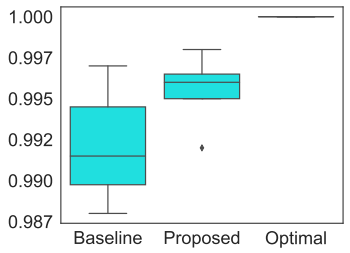

Fig. 8: Distributions of TAR@FAR=0.3 across subgroups. Less of a span between the $\mathrm{max} / \mathrm{min}$ and a boost in performances are shown, comparing the proposed (middle) to the baseline (left).

TABLE 3: TAR at intervals of FAR. TAR scores for a global threshold (top), the proposed debiasing transformation (middle), optimal threshold (bottom): the higher, the better. The standard deviation from the average is shown to demonstrate the standard error comparing the reported (i.e., average) to the subgroup-specific (i.e., actual) scores (Fig. 8)-the proposed recovers most of the loss from using a global threshold rather than a per-subgroup threshold.

\begin{tabular}{cccccc} 
FAR & 0.3 & 0.1 & 0.01 & 0.001 & 0.0001 \\
\hline \multirow{4}{*}{ AF } & 0.990 & 0.867 & 0.516 & 0.470 & 0.465 \\
& 0.996 & 0.874 & 0.521 & 0.475 & 0.470 \\
& 1.000 & 0.882 & 0.524 & 0.478 & 0.474 \\
& 0.994 & 0.883 & 0.529 & 0.482 & 0.477 \\
AM & 0.996 & 0.886 & 0.531 & 0.484 & 0.479 \\
& 1.000 & 0.890 & 0.533 & 0.486 & 0.482 \\
& 0.991 & 0.870 & 0.524 & 0.479 & 0.473 \\
BF & 0.995 & 0.875 & 0.527 & 0.481 & 0.476 \\
& 1.000 & 0.879 & 0.530 & 0.484 & 0.480 \\
& 0.992 & 0.881 & 0.526 & 0.480 & 0.474 \\
BM & 0.995 & 0.886 & 0.529 & 0.483 & 0.478 \\
& 1.000 & 0.891 & 0.532 & 0.485 & 0.480 \\
& 0.996 & 0.881 & 0.532 & 0.486 & 0.481 \\
IF & 0.998 & 0.883 & 0.533 & 0.487 & 0.483 \\
& 1.000 & 0.884 & 0.534 & 0.488 & 0.484 \\
& 0.997 & 0.895 & 0.533 & 0.485 & 0.479 \\
IM & 0.998 & 0.897 & 0.534 & 0.486 & 0.480 \\
& 1.000 & 0.898 & 0.535 & 0.486 & 0.481 \\
& 0.988 & 0.878 & 0.517 & 0.469 & 0.464 \\
WF & 0.992 & 0.884 & 0.522 & 0.472 & 0.468 \\
& 1.000 & 0.894 & 0.526 & 0.478 & 0.474 \\
& 0.989 & 0.896 & 0.527 & 0.476 & 0.470 \\
WM & 0.996 & 0.901 & 0.530 & 0.479 & 0.474 \\
& 1.000 & 0.910 & 0.535 & 0.483 & 0.478 \\
\hline \multirow{4}{*}{ Avg. } & 0.992 & 0.881 & 0.526 & 0.478 & 0.473 \\
& 0.998 & 0.886 & 0.528 & 0.481 & 0.476 \\
& 1.000 & 0.891 & 0.531 & 0.483 & 0.479
\end{tabular}

that is more globally understood, and more comprehensible with respect to the use-case. A prime example is to share the error rate per number correct (e.g., falsely classify one in ten thousand). For instance, claiming that a system predicts $1 \mathrm{FP}$ per 10,000 predictions. However, such an approximation can be hazardous, for it is inherently misleading. To demonstrate this, we ask the following questions. Does this claim hold true for different demographics? Does this rating depend on the types of faces - does it hold for all males or all females? Thus, if we set our system according to a desired FAR, would the claim be fair regardless of demographics (i.e., subgroups) of population.

The aforementioned questions were central to our previous work [11]. We found the answer to these questions to be clear No, the report FAR is not true when analyzed per subgroup. We found when comparing the False Acceptance Rate (FAR) values (i.e., the reported-to-the-actual), the values drastically deviate from the reported average when the score threshold is fixed for all subgroups. Furthermore, demographic-specific thresholds, meaning an assumption that demographic information is known prior, proved to mitigate the problem. However, prior knowledge of demographic, although plausible (e.g., identifying a known subject on a black list), it is a strong assumption that limits the practical uses for which it could be deployed. To extend our prior work, we propose a debiasing scheme to reduce the differences between the reported and actual. We set out to make the claim in reported false rates to be fair across all involved demographics.

\subsubsection{Metrics and settings}

TAR and FAR are used to examine the trade-off in the confusions that is dependent on the choice of threshold discussed earlier. Specifically, we look at actual TAR scores as a function of desired FAR. Furthermore, we compute the following metric, the percent difference of the actual and reported FAR values (i.e., an average score is targeted). So we ask, how well do the different subgroups compare to that average? Specifically,

$$
\% \operatorname{Error}(l)=\left(\frac{\operatorname{FAR}(l)_{\text {reported }}-\operatorname{FAR}(l)_{\text {actual }}}{\operatorname{FAR}(l)_{\text {reported }}}\right) * 100 \%
$$

The reported results are the results averaged across subgroups. Then, for individual subgroups, the actual result, which tends to differ meaningfully from the mean result (i.e., the reported results), is analyzed independently. Hence, there is a gap between reported and actual, which we show in Fig. 7 using the percent difference (i.e., 100\%* (actual - reported)/reported). Note that the percent difference takes on negative values when reported $>$ actual, which translates to the average performance of a subgroup being worse than the average performance.

\subsubsection{Analysis}

The proposed balance results, and significantly boosts the TAR at FAR. The percent difference between reported and actual FAR scores leads to a more fair representation, especially at high FAR. Fig. 7 demonstrates the spread of scores for the various subgroups using the baseline, the proposed, and the optimal threshold at 0.3 FAR. Note that there the standard deviation of TAR using the Baseline approach is very high and that this is mitigated using the proposed scheme. The proposed has thus boosted performance: improved the overall rating, and reduced the variances (Table 3).

Fig. 7 can be interpreted as a practical use case. The reported FPR implies that the system has its threshold set to yield a measure depicting the number of $\mathrm{TP}$ a verification system reports on average before making an error (i.e., the number of TP before an FP is expected). The far-right (i.e., 1e-4) claims that 1 in 10,000 is incorrectly matched. Again, in practice, a predetermined FPR for a verification system is set via a trade-off threshold value (i.e., $\theta$ ) that controls the level of sensitivity: decreasing the score threshold decreases the FPR (Fig. 2). Fig. 7 then shows, for a set of faces with equal representation for all subgroups, the difference between the reported and actual on the subgroup level.

The baseline shows a $200 \%$ increase for $\mathrm{WM}$ for $\mathrm{FPR}=1 \mathrm{e}-$ 4. Compared to the reported performance that expects an FP per 10,000 TP, WM doubles this. This means that for a population consisting of only WM, the actual result would be 1 per 20,000 TP.

As mentioned, the direction (i.e., \pm ) represents whether the difference is an improvement. A negative \%-difference indicates a drop in performance when compared to the reported. For instance, AF with a $-25 \%$ difference for the baseline at an overall average FPR of 1 in 10,000 implies that if the population of samples consists of only AF subjects, then the FPR for the given $\theta$ chosen 


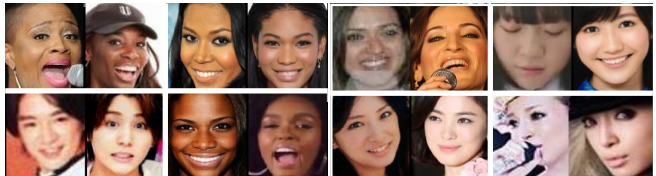

(a) $\mathrm{TN}$.

(b) TP.

Fig. 9: Hard sample pairs. Fooled the baseline but corrected by the proposed: true negatives (a) and true positives (b).

for the claim of 1 in 10,000 would be 1 in 7,500. Thus, a consumer that is expecting a specific FPR would only ever match this value when the sample population has the same distribution in samples per subgroup as that of the validation data for which $\theta$ was found.

The percent difference can be removed via an optimal threshold. However, the assumption that subgroup-specific thresholds can be determined from validation sets that are separated by subgroup. Also, at test time, the optimal solution assumes prior knowledge of the subgroup to which the sample of interest belongs. Although the method was proof in principle, both of the assumptions are impractical for most use-cases. Hence, the proposed feature transformations reduce the percent differences from the original features (i.e., the baseline). The proposed and the optimal are the middle and right plots, respectively (Fig. 7).

Fig. 9 shows several hard positives and negatives that were incorrectly matched by the baseline but correctly identified by the proposed. These samples had scores closest to the global threshold (i.e., score boundary). Notice the quality of at least one face per pair is low-resolution; additionally, extreme pose differences between faces of each pair also are common. These challenges are overcome by the proposed scheme - mitigating the issue of bias boosts results, and there displayed are several of the pairs that went from falsely being rejected to correctly being accepted.

\subsection{Privacy preserving experiment}

As mentioned, our objective was two-fold. First, we aimed to preserve identity information while debiasing the facial features, as demonstrated in the prior experiment. But then, secondly, our objective function injected the reverse gradient as part of the loss to force the embedding to be unable to classify subgroups (i.e., demographics). In other words, another benefit of the proposed debiasing scheme is that it rids the facial features of demographic information. This, in itself, is of interest in problems of privacy and protection - ideally, face features, which often are the only representation of face information available at the system level, will not include attribute information, like gender or ethnicity. Hence, we prohibit subgroup classifiers the ability to learn from the features (i.e., reduce demographic information in the features).

We train a multi-layered perceptron (MLP) to classify subgroups on top of the features to show the extent that the proposed removes subgroup information. We can then measure the amount of information present in the face representation [33].

The MLP designed in Keras as follows: three fully-connected layers of size 512, 512, and 256 fed into the output fullyconnected layer (i.e., size 8 for each subgroup class). The first three layers were separated by ReLU activation and dropout [91] (i.e., probability of 0.5 ), while only dropout (again, 0.5 ) was place prior to the output softmax layer. A categorical cross-entropy loss with Adam [92] set with a learning rate of 1e-3 was used to train.

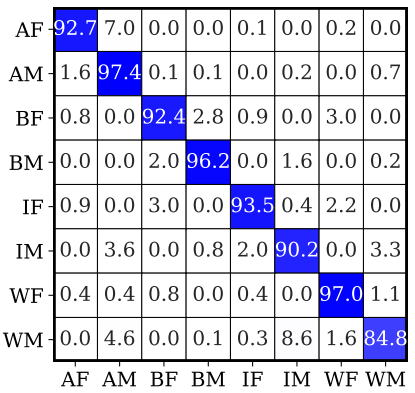

(a) Baseline classifier.

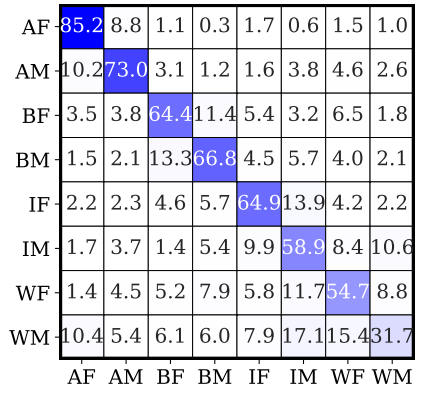

(b) Our classifier.
Fig. 10: Subgroup confusion matrix. Comparison of accuracy in classifying and misclassifying the subgroups. Notice the (b) performs significantly worse than (a) as intended.

\subsubsection{Metrics and settings}

We examine the overall accuracy of the subgroup classifiers via a confusion matrix. Specifically, we will look at how often each subgroup was predicted correctly and, when incorrect, the percentage it was mistaken for the others. The confusion was generated by averaging across the five folds. For all subgroup classifiers, the threshold that yielded the top performance on the training folds was then used on the test fold.

Also, we measure precision and recall. Precision, a measure of time correct when the prediction is assumed to be true, is calculated as $\mathrm{P}(l)=\frac{\mathrm{TP}}{\mathrm{TP}+\mathrm{FP}}$, which we average across subgroups $l \in L$. The recall $\mathrm{R}$, which is the ratio of the number of predicted-to-total positive samples, is found as $\mathrm{R}(l)=\frac{\mathrm{TP}}{\mathrm{TP}+\mathrm{FN}}$. This complements the confusion by allowing the specificity and sensitivity of the subgroups to also be examined. Nonetheless, there are inherent trade-offs between $\mathrm{P}$ and $\mathrm{R}$. This motivates the $F_{1}$-score [93], as the harmonic mean of $\mathrm{P}$ and $\mathrm{R}, \mathrm{F}_{1}=2 * \frac{P * R}{P+R}$.

\subsubsection{Analysis}

We showed the preservation of identity knowledge (Table 3), and now we show the other benefits of privacy. The results confirm that the privacy-preserving claim is accurate, leading to a $30 \%$ drop in the ability to predict gender and ethnicity from the features (Table 4). Hence, the predictive power of all subgroups dropped significantly. The drop in performance suffices to make the claim that the predictions are now unreliable. It is interesting to note that it hindered the subgroups for which the baseline was most in favor the most from the debias scheme. WM and WF drop the most, while the AM and AF drop the least. All the while, the same

TABLE 4: Subgroup classification results. The baseline and proposed are on the left and right columns, respectively. Note that the columns on the right have lower scores as intended.

\begin{tabular}{|c|c|c|c|c|c|c|}
\hline \multirow[b]{2}{*}{$\mathrm{AF}$} & \multicolumn{2}{|c|}{ Precision } & \multicolumn{2}{|c|}{ Recall } & \multicolumn{2}{|c|}{ F1 } \\
\hline & 0.962 & 0.734 & 0.927 & 0.852 & 0.943 & 0.788 \\
\hline AM & 0.864 & 0.707 & 0.974 & 0.730 & 0.915 & 0.717 \\
\hline $\mathrm{BF}$ & 0.940 & 0.655 & 0.924 & 0.644 & 0.932 & 0.647 \\
\hline BM & 0.961 & 0.644 & 0.962 & 0.668 & 0.961 & 0.653 \\
\hline IF & 0.961 & 0.641 & 0.935 & 0.649 & 0.948 & 0.644 \\
\hline IM & 0.898 & 0.519 & 0.902 & 0.589 & 0.898 & 0.550 \\
\hline WF & 0.934 & 0.554 & 0.970 & 0.547 & 0.951 & 0.549 \\
\hline WM & 0.943 & 0.524 & 0.848 & 0.317 & 0.892 & 0.392 \\
\hline & 0.933 & 0.622 & 0.930 & 0.624 & 0.930 & 0.617 \\
\hline
\end{tabular}




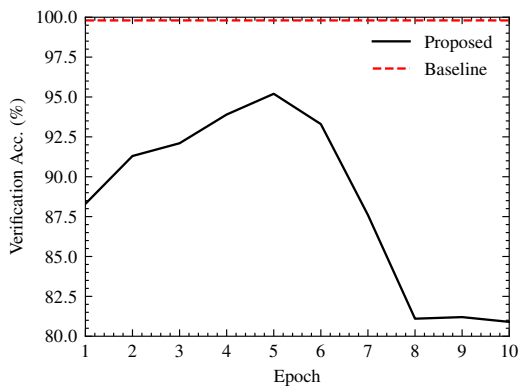

Fig. 11: Accuracy on LFW. The proposed nears the performance of the baseline of $99.8 \%$, with its top score of $95.2 \%$. (Used https://github.com/garrettj403/SciencePlots/ to generate the plot.)

trends in confusion propagate from the baseline to the proposed results (e.g., WM mostly confuses as IM initially, and then again with the proposed). The same holds for cases of the opposite sex.

Next, we examine the confusions for the different subgroups before and after debiasing the face features (Fig. 10). As established, the baseline contains more subgroup knowledge- a model can learn on top. When trained and evaluated on BFW, the baseline performs better on F subgroups. This differs from the norm, where $\mathrm{M}$ is the majority of the data. On the contrary, the $\mathrm{WM}$ is inferior in performance to all subgroups in either case.

\subsection{Ablation study}

To check the effectiveness of the proposed, we train M on the BFW dataset and deploy it on the well-known LFW benchmark. We note that the training dataset we employ is significantly smaller than that used by SOTA networks trained to achieve high performance on LFW, employing the MS1MV2 dataset, which contains $5.8 \mathrm{M}$ images of $85 \mathrm{k}$ identities. Even though we initialize our network starting with features learned on MS1MV2, we train on a small dataset of $20 \mathrm{k}$ images of 800 subjects, two orders of magnitude smaller. The current SOTA with $99.8 \%$ verification accuracy, while the proposed scheme reaches its best score of $95.2 \%$ after five epochs before dropping off and then leveling out around $81 \%$ (Fig. 11). The unbalanced data hinders the benefits of privacy and debiasing (i.e., LFW comprises about 85\% WM). Furthermore, we optimized $\mathrm{M}$ by choosing the epoch with the best performance before the drop-off. Future steps could improve the proposed when transferring to unbalanced sets to detect the optimal settings.

\section{CONCLUSION AND FUTURE WORK}

We show a bias for subgroups in FR caused by the selection of a single threshold. Previously, we proposed a subgroupspecific threshold as a solution for the case in which knowledge of subgroup information is accessible prior. Inspired by works in feature alignment and domain adaptation, we introduce a scheme to mitigate the bias present in face features. We learn a lower-dimensional mapping that preserves identity and removes the knowledge of the subgroups. With the proposed, the FR features balance the performance across subgroups while boosting the overall accuracy. Using a single threshold, we reduce the difference between actual and average (i.e., reported) performance across subgroups. The resulting features hold reduced knowledge of subgroups, increasing privacy concerning demographics (i.e., gender and ethnicity). BFW and source code are publicly available for use in research and practice.
Looking ahead, open problems in the experimental settings and practice remain. For instance, gender labels are boolean, which is a gross approximation of individual sexuality - better represented as real, continuous values [60]. Also, the definitions of race and ethnicity are loosely defined. The US Census Bureau allows an individual to choose their race (https://www.census.gov). Finergrained subgroups would be another means to improve the experiments (e.g., Indians from North India versus South India, or Africans versus African Americans, or distinct groups across Africa). The labels and definitions we use here are sufficient to demonstrate the value of our work and move towards providing a test-bed to produce PR results fairly.

\section{REFERENCES}

[1] A. Acien, A. Morales, R. Vera-Rodriguez, I. Bartolome, and J. Fierrez, "Measuring the gender and ethnicity bias in deep models for face recognition," in Progress in Pattern Recognition, Image Analysis, Computer Vision, and Applications, R. Vera-Rodriguez, J. Fierrez, and A. Morales, Eds. Springer International Publishing, 2019. 1

[2] L. Anne Hendricks, K. Burns, K. Saenko, T. Darrell, and A. Rohrbach, "Women also snowboard: Overcoming bias in captioning models," in Proceedings of the European Conference on Computer Vision, 2018. 1

[3] M. Wang, W. Deng, J. Hu, J. Peng, X. Tao, and Y. Huang, "Race faces inthe-wild: Reduce bias by deep unsupervised domain adaptation," CoRR arXiv:1812.00194, 2019. 1, 3, 4

[4] C. Lazo, "Towards engineering ai software for fairness: A framework to help design fair, accountable and transparent algorithmic decisionmaking systems," TU Delft Library, 2020. 1

[5] I. D. Raji and J. Buolamwini, "Actionable auditing: Investigating the impact of publicly naming biased performance results of commercial ai products," in Proceedings of the 2019 AAAI/ACM Conference on AI, Ethics, and Society, 2019, pp. 429-435. 1

[6] P. Drozdowski, C. Rathgeb, A. Dantcheva, N. Damer, and C. Busch, "Demographic bias in biometrics: A survey on an emerging challenge," IEEE Transactions on Technology and Society, 2020. 1, 2, 3

[7] J. Deng, J. Guo, N. Xue, and S. Zafeiriou, "Arcface: Additive angular margin loss for deep face recognition," in IEEE Conference on Computer Vision and Pattern Recognition (CVPR), 2019. 1, 2

[8] W. Liu, Y. Wen, Z. Yu, M. Li, B. Raj, and L. Song, "Sphereface: Deep hypersphere embedding for face recognition," in IEEE Conference on Computer Vision and Pattern Recognition (CVPR), 2017. 1, 2

[9] F. Wang, J. Cheng, W. Liu, and H. Liu, "Additive margin softmax for face verification," IEEE Signal Processing Letters, 2018. 1, 7

[10] H. Wang, Y. Wang, Z. Zhou, X. Ji, D. Gong, J. Zhou, Z. Li, and W. Liu, "Cosface: Large margin cosine loss for deep face recognition," in IEEE Conference on Computer Vision and Pattern Recognition, 2018. 1

[11] J. P. Robinson, G. Livitz, Y. Henon, C. Qin, Y. Fu, and S. Timoner, "Face recognition: too bias, or not too bias?" in Computer Vision and Pattern Recognition Workshop, 2020, pp. 0-1. 1, 2, 4, 5, 7, 8

[12] K. W. Bowyer, "Face recognition technology: security versus privacy," IEEE Technology and society magazine, pp. 9-19, 2004. 1

[13] Y. Taigman, M. Yang, M. Ranzato, and L. Wolf, "Deepface: Closing the gap to human-level performance in face verification," in IEEE Conference on Computer Vision and Pattern Recognition (CVPR), 2014. 2

[14] O. M. Parkhi, A. Vedaldi, and A. Zisserman, "Deep face recognition," in British Machine Vision Conference (BMVC), 2015. 2

[15] Y. Wen, K. Zhang, Z. Li, and Y. Qiao, "A discriminative feature learning approach for deep face recognition," in European Conference on Computer Vision, 2016, pp. 499-515. 2

[16] Y. Duan, J. Lu, and J. Zhou, "Uniformface: Learning deep equidistributed representation for face recognition," in IEEE Conference on Computer Vision and Pattern Recognition (CVPR), 2019, pp. 3415-3424. 2

[17] Z. Wang, G. Wang, B. Huang, Z. Xiong, Q. Hong, H. Wu, P. Yi, K. Jiang, N. Wang, Y. Pei et al., "Masked face recognition dataset and application," CoRR arXiv:2003.09093, 2020. 2

[18] G. Guo and N. Zhang, "A survey on deep learning based face recognition," Computer Vision and Image Understanding, 2019. 2

[19] I. Masi, Y. Wu, T. Hassner, and P. Natarajan, "Deep face recognition: A survey," in 201831 st SIBGRAPI conference on graphics, patterns and images (SIBGRAPI). IEEE, 2018, pp. 471-478. 2

[20] A. Khalil, S. G. Ahmed, A. M. Khattak, and N. Al-Qirim, "Investigating bias in facial analysis systems: A systematic review," IEEE Access, vol. 8, pp. 130751-130761, 2020. 2 
[21] V. Muthukumar, T. Pedapati, N. Ratha, P. Sattigeri, C.-W. Wu, B. Kingsbury, A. Kumar, S. Thomas, A. Mojsilovic, and K. R. Varshney, "Understanding unequal gender classification accuracy from face images," CoRR arXiv:1812.00099, 2018. 2

[22] J. Buolamwini and T. Gebru, "Gender shades: Intersectional accuracy disparities in commercial gender classification," in Conference on fairness, accountability and transparency. PMLR, 2018. 2

[23] P. Grother, M. Ngan, and K. Hanaoka, "Face recognition vendor test part 3: Demographic effects," 2019-12-19 2019. 2

[24] G. Balakrishnan, Y. Xiong, W. Xia, and P. Perona, "Towards causal benchmarking of bias in face analysis algorithms," CoRR arXiv:2007.06570, 2020. 2

[25] M. Georgopoulos, J. Oldfield, M. A. Nicolaou, Y. Panagakis, and M. Pantic, "Enhancing facial data diversity with style-based face aging," in Computer Vision and Pattern Recognition Workshop, 2020. 2

[26] V. Muthukumar, T. Pedapati, N. Ratha, P. Sattigeri, C.-W. Wu, B. Kingsbury, A. Kumar, S. Thomas, A. Mojsilović, and K. R. Varshney, "Color-theoretic experiments to understand unequal gender classification accuracy from face images," in Computer Vision and Pattern Recognition Workshop, 2019, pp. 2286-2295. 2

[27] P. Terhörst, J. N. Kolf, N. Damer, F. Kirchbuchner, and A. Kuijper, "Postcomparison mitigation of demographic bias in face recognition using fair score normalization," CoRR arXiv:2002.03592, 2020. 2

[28] I. Serna, A. Peña, A. Morales, and J. Fierrez, "Insidebias: Measuring bias in deep networks and application to face gender biometrics," CoRR arXiv:2004.06592, 2020. 2

[29] V. Albiero, K. KS, K. Vangara, K. Zhang, M. C. King, and K. W. Bowyer, "Analysis of gender inequality in face recognition accuracy," in Computer Vision and Pattern Recognition Workshop, 2020, pp. 81-89. 2

[30] A. Das, A. Dantcheva, and F. Bremond, "Mitigating bias in gender, age and ethnicity classification: a multi-task convolution neural network approach," in European Conference on Computer Vision, 2018. 2

[31] N. Srinivas, K. Ricanek, D. Michalski, D. S. Bolme, and M. King, "Face recognition algorithm bias: Performance differences on images of children and adults," in Computer Vision and Pattern Recognition Workshop, 2019. 2

[32] S. Nagpal, M. Singh, R. Singh, M. Vatsa, and N. Ratha, "Deep learning for face recognition: Pride or prejudiced?" CoRR arXiv:1904.01219, 2019. 2

[33] A. Acien, A. Morales, R. Vera-Rodriguez, I. Bartolome, and J. Fierrez, "Measuring the gender and ethnicity bias in deep models for face recognition," in Iberoamerican Congress on Pattern Recognition. Springer, 2018. 2, 9

[34] S. Gong, X. Liu, and A. K. Jain, "Debface: De-biasing face recognition," CoRR arXiv:1911.08080, 2019. 2

[35] A. V. Savchenko, "Efficient facial representations for age, gender and identity recognition in organizing photo albums using multi-output convnet," PeerJ Computer Science, vol. 5, p. e197, 2019. 2

[36] S. Nagpal, M. Singh, R. Singh, and M. Vatsa, "Attribute aware filterdrop for bias invariant classification," in Computer Vision and Pattern Recognition Workshop, June 2020. 2

[37] T. Sixta, J. Junior, C. Jacques, P. Buch-Cardona, E. Vazquez, and S. Escalera, "Fairface challenge at eccv 2020: Analyzing bias in face recognition," CoRR arXiv:2009.07838, 2020. 2

[38] A. Peña, I. Serna, A. Morales, and J. Fierrez, "Bias in multimodal ai: Testbed for fair automatic recruitment," arXiv:2004.07173, 2020. 2

[39] _ _Faircvtest demo: Understanding bias in multimodal learning with testbed in fair automatic recruitment," CoRR arXiv:2009.07025, 2020. 2

[40] P. Terhörst, N. Damer, F. Kirchbuchner, and A. Kuijper, "Suppressing gender and age in face templates using incremental variable elimination," in 2019 International Conference on Biometrics (ICB), 2019, pp. 1-8. 2

[41] B. Sadeghi and V. N. Boddeti, "Imparting fairness to pre-trained biased representations," in Computer Vision and Pattern Recognition Workshop, June 2020. 2

[42] M. Wang and W. Deng, "Mitigating bias in face recognition using skewness-aware reinforcement learning," in IEEE Conference on Computer Vision and Pattern Recognition (CVPR), 2020. 2

[43] P.-M. Law, S. Malik, F. Du, and M. Sinha, "Designing tools for semiautomated detection of machine learning biases: An interview study," CoRR arXiv:2003.07680, 2020. 2

[44] C. Drummond, R. C. Holte et al., "C4. 5, class imbalance, and cost sensitivity: why under-sampling beats over-sampling," in Workshop on learning from imbalanced datasets II. Citeseer, 2003. 2

[45] M. Oquab, L. Bottou, I. Laptev, and J. Sivic, "Learning and transferring mid-level image representations using convolutional neural networks," in IEEE Conference on Computer Vision and Pattern Recognition (CVPR), 2014, pp. 1717-1724. 2
[46] E. M. Rudd, M. Günther, and T. E. Boult, "Moon: A mixed objective optimization network for the recognition of facial attributes," in European Conference on Computer Vision. Springer, 2016, pp. 19-35. 3

[47] C. Huang, Y. Li, C. L. Chen, and X. Tang, "Deep imbalanced learning for face recognition and attribute prediction," IEEE Transactions on Pattern Analysis and Machine Intelligence (TPAMI), 2019. 3

[48] T. Wang, J. Zhao, M. Yatskar, K.-W. Chang, and V. Ordonez, "Balanced datasets are not enough: Estimating and mitigating gender bias in deep image representations," in IEEE International Conference on Computer Vision (ICCV), 2019, pp. 5310-5319. 3

[49] H. He and E. A. Garcia, "Learning from imbalanced data," IEEE Transactions on knowledge and data engineering, 2009. 3

[50] H. He and Y. Ma, Imbalanced learning: foundations, algorithms, and applications. John Wiley \& Sons, 2013. 3

[51] B. Krawczyk, "Learning from imbalanced data: open challenges and future directions," Progress in Artificial Intelligence, 2016. 3

[52] T. Xu, J. White, S. Kalkan, and H. Gunes, "Investigating bias and fairness in facial expression recognition," CoRR arXiv:2007.10075, 2020. 3

[53] V. Albiero, K. Zhang, and K. Bowyer, "How gender balance in training data affect face recognition accuracy?" arXiv:2002.02934, 2020. 3

[54] Y. Guo, L. Zhang, Y. Hu, X. He, and J. Gao, "Ms-celeb-1m: A dataset and benchmark for large-scale face recognition," in European Conference on Computer Vision, 2016. 3, 7

[55] F. Schroff, D. Kalenichenko, and J. Philbin, "Facenet: A unified embedding for face recognition and clustering," in IEEE Conference on Computer Vision and Pattern Recognition (CVPR), 2015, pp. 815-823. 3

[56] Q. Cao, L. Shen, W. Xie, O. M. Parkhi, and A. Zisserman, "Vggface2: A dataset for recognising faces across pose and age," in IEEE International Conference on Automatic Face and Gesture Recognition, 2018. 3, 5

[57] B. Maze, J. Adams, J. A. Duncan, N. Kalka, T. Miller, C. Otto, A. K. Jain, W. T. Niggel, J. Anderson, J. Cheney et al., "Iarpa janus benchmarkc: Face dataset and protocol," in 2018 International Conference on Biometrics (ICB). IEEE, 2018, pp. 158-165. 3

[58] I. Hupont and C. Fernández, "Demogpairs: Quantifying the impact of demographic imbalance in deep face recognition," in Conference on Automatic Face and Gesture Recognition. IEEE, 2019. 3, 4

[59] K. Kärkkäinen and J. Joo, "Fairface: Face attribute dataset for balanced race, gender, and age," CoRR arXiv:1908.04913, 2019. 3, 4

[60] M. Merler, N. Ratha, R. S. Feris, and J. R. Smith, "Diversity in faces," CoRR arXiv:1901.10436, 2019. 3, 10

[61] D. Yi, Z. Lei, S. Liao, and S. Z. Li, "Learning face representation from scratch," CoRR arXiv:1411.7923, 2014. 3

[62] Z. Ding, S. Li, M. Shao, and Y. Fu, "Graph adaptive knowledge transfer for unsupervised domain adaptation," in ECCV, 2018. 3

[63] X. Peng, B. Usman, N. Kaushik, J. Hoffman, D. Wang, and K. Saenko, "Visda: The visual domain adaptation challenge," CoRR arXiv:1710.06924, 2017. 3

[64] K. Saito, D. Kim, S. Sclaroff, T. Darrell, and K. Saenko, "Semi-supervised domain adaptation via minimax entropy," CoRR arXiv:1904.06487, 2019. 3

[65] R. Shu, H. H. Bui, H. Narui, and S. Ermon, "A dirt-t approach to unsupervised domain adaptation," CoRR arXiv:1802.08735, 2018. 3

[66] B. Sun and K. Saenko, "Subspace distribution alignment for unsupervised domain adaptation." in $B M V C, 2015.3$

[67] M. Long, J. Wang, G. Ding, J. Sun, and P. S. Yu, "Transfer feature learning with joint distribution adaptation," in ICCV, 2013. 3

[68] B. Gong, Y. Shi, F. Sha, and K. Grauman, "Geodesic flow kernel for unsupervised domain adaptation," in CVPR, 2012. 3

[69] R. Gopalan, R. Li, and R. Chellappa, "Domain adaptation for object recognition: An unsupervised approach," in ICCV, 2011. 3

[70] Y. Ganin, E. Ustinova, H. Ajakan, P. Germain, H. Larochelle, F. Laviolette, M. Marchand, and V. Lempitsky, "Domain-adversarial training of neural networks," JMLR, vol. 17, no. 1, 2016. 3

[71] E. Tzeng, J. Hoffman, K. Saenko, and T. Darrell, "Adversarial discriminative domain adaptation," in CVPR, 2017. 3

[72] B. H. Zhang, B. Lemoine, and M. Mitchell, "Mitigating unwanted biases with adversarial learning," in Proceedings of the AAAI/ACM Conference on AI, Ethics, and Society, 2018, pp. 335-340. 3

[73] P. Dhar, J. Gleason, H. Souri, C. D. Castillo, and R. Chellappa, "An adversarial learning algorithm for mitigating gender bias in face recognition," CoRR arXiv:2006.07845, 2020. 3

[74] V. Mirjalili, S. Raschka, and A. Ross, "Gender privacy: An ensemble of semi adversarial networks for confounding arbitrary gender classifiers,' in 2018 IEEE 9th International Conference on Biometrics Theory Applications and Systems (BTAS), 2018, pp. 1-10. 3

[75] J. Hamm, "Minimax filter: Learning to preserve privacy from inference attacks," The Journal of Machine Learning Research, 2017. 5 
[76] M. Bertran, N. Martinez, A. Papadaki, Q. Qiu, M. Rodrigues, G. Reeves, and G. Sapiro, "Adversarially learned representations for information obfuscation and inference," in International Conference on Machine Learning (ICML). PMLR, 2019, pp. 614-623. 5

[77] P. C. Roy and V. N. Boddeti, "Mitigating information leakage in image representations: A maximum entropy approach," in IEEE Conference on Computer Vision and Pattern Recognition (CVPR), 2019. 5

[78] Z. Wu, H. Wang, Z. Wang, H. Jin, and Z. Wang, "Privacy-preserving deep action recognition: An adversarial learning framework and a new dataset," IEEE Transactions on Pattern Analysis and Machine Intelligence (TPAMI), pp. 1-1, 2020. 5

[79] Z. Wu, Z. Wang, Z. Wang, and H. Jin, "Towards privacy-preserving visual recognition via adversarial training: A pilot study," in European Conference on Computer Vision, 2018, pp. 606-624. 5

[80] Y. Ganin and V. Lempitsky, "Unsupervised domain adaptation by backpropagation," in International Conference on Machine Learning (ICML). PMLR, 2015, pp. 1180-1189. 6, 7

[81] A. Othman and A. Ross, "Privacy of facial soft biometrics: Suppressing gender but retaining identity," in Computer Vision - ECCV 2014 Workshops, L. Agapito, M. M. Bronstein, and C. Rother, Eds. Cham: Springer International Publishing, 2015, pp. 682-696. 5

[82] S. Guo, T. Xiang, and X. Li, "Towards efficient privacy-preserving face recognition in the cloud," Signal Processing, pp. $320-328,2019.5$

[83] Z. Ma, Y. Liu, X. Liu, J. Ma, and K. Ren, "Lightweight privacypreserving ensemble classification for face recognition," IEEE Internet of Things Journal, vol. 6, no. 3, pp. 5778-5790, 2019. 5

[84] A. Ambekar, C. Ward, J. Mohammed, S. Male, and S. Skiena, "Nameethnicity classification from open sources," in Proceedings of SIGKDD conference on Knowledge Discovery and Data Mining, 2009. 5

[85] S. Fu, H. He, and Z.-G. Hou, "Learning race from face: A survey," IEEE Transactions on Pattern Analysis and Machine Intelligence (TPAMI), 2014. 5

[86] G. Levi and T. Hassner, "Age and gender classification using convolutional neural networks," in Computer Vision and Pattern Recognition Workshop, 2015, pp. 34-42. 5

[87] H. V. Nguyen and L. Bai, "Cosine similarity metric learning for face verification," in Asian Conference on Computer Vision (ACCV). Springer, 2010, pp. 709-720. 5

[88] G. B. Huang, M. Ramesh, T. Berg, and E. Learned-Miller, "Labeled faces in the wild: A database for studying face recognition in unconstrained environments," U. of Massachusetts, Amherst, Tech. Rep., 2007. 6, 7

[89] K. Zhang, Z. Zhang, Z. Li, and Y. Qiao, "Joint face detection and alignment using multitask cascaded convolutional networks," Signal Processing Letters, 2016. 7

[90] F. Wang, X. Xiang, J. Cheng, and A. L. Yuille, "Normface: L2 hypersphere embedding for face verification," in ACM Conference on Multimedia, 2017, pp. 1041-1049. 7

[91] N. Srivastava, G. Hinton, A. Krizhevsky, I. Sutskever, and R. Salakhutdinov, "Dropout: a simple way to prevent neural networks from overfitting," The journal of machine learning research, vol. 15, no. 1, pp. 1929-1958, 2014. 9

[92] D. P. Kingma and J. Ba, "Adam: A method for stochastic optimization," CoRR arXiv:1412.6980, 2014. 9

[93] L. A. Jeni, J. F. Cohn, and F. De La Torre, "Facing imbalanced datarecommendations for the use of performance metrics," in Humaine association conference on affective computing and intelligent interaction. IEEE, 2013, pp. 245-251. 9

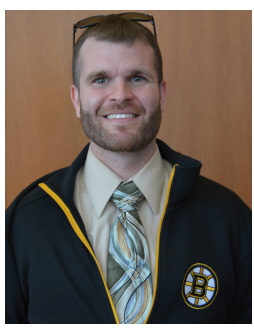

Joseph P Robinson Received a B.S. in ECE (2014) and Ph.D. in CE (2020) at Northeastern University, where he was also part-time faculty: designed and taught Data Analytics (2020 Best Teacher). Research is in applied machine vision, with emphasis on faces, deep learning, MM, big data. Previously, led a team to TRECVid debut (MED, 3rd-place). Built many image and video datasets- most notably FIW. Organized 2020 FG conference, various workshops and challenges (e.g., NECV, RFIW, AMFG, FacesMM), tutorials (MM, CVPR, FG), PC (e.g., CVPR, FG, MIRP, MMEDIA, AAAI, ICCV), reviewer (e.g., IEEE TBioCAS, TIP, TPAMI), and positions like President of IEEE@NEU (grad student adviser) and Relations Officer of IEEE SAC R1. Completed: NSF REUs (2010 \& 2011); interned at Analogic Corporation (2012) and BBN Tech. (2013), MIT Lincoln Labs (2014), System \& Tech. Research (2016 \& 2017), Snap Inc. (2018), ISM (2019). Now, works on robots at Vicarious Surgical (2021).

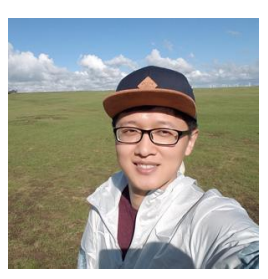

Can Qin Received the B.E. from the School of Microelectronics, Xidian University, China (2018), and is pursuing a Ph.D. at the Department of Electrical and Computer Engineering, Northeastern University, under Dr. Yun Raymond Fu. His research focus spans transfer learning, semi-supervised learning, and deep learning in broad. He received awards for the Best Paper Award of the Real-World Recognition from Low-Quality Images and Videos workshop at 2019 ICCV. Additionally, he has published at top-tier conferences (i.e., NeurIPS, AAAI, ECCV).

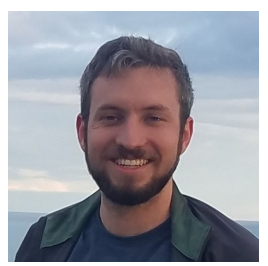

Yann Henon received a B.S. and an M.S from Monash University in Melbourne, Australia. His research has focused on medical imaging and has been published in top journals including Developmental Cell, Journal of Applied Physiology and RSC Advances.

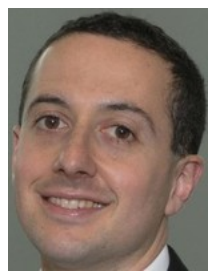

Samson Timoner B.S. in Applied Physics from Caltech (1997); Ph.D. in Electrical Engineering and Computer Science From MIT (2003). He has organized numerous workshops including CVPR View, CVPR ProCams, the New Computer Vision Workshop. He has started two computer vision companies. He is an organizer of the computer vision community in Boston, bringing researchers and entrepreneurs together. $\mathrm{He}$ is currently Principal Scientist at ISM focusing on facial detection and recognition algorithms.

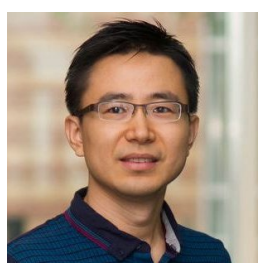

Yun Fu (S'07-M'08-SM'11-F'19) received the B.Eng. degree in information engineering and the M.Eng. degree in pattern recognition and intelligence systems from Xi'an Jiaotong University, China, respectively, and the M.S degree in statistics and the Ph.D. degree in electrical and computer engineering from the University of Illinois at Urbana-Champaign, respectively. He is an interdisciplinary faculty member affiliated with College of Engineering and the College of Computer and Information Science at Northeastern University since 2012. His research interests are Machine Learning, Computational Intelligence, Big Data Mining Computer Vision, Pattern Recognition, and Cyber-Physical Systems. $\mathrm{He}$ has extensive publications in leading journals, books/book chapters and international conferences/workshops. He serves as associate editor, chairs, PC member and reviewer of many top journals and international conferences/workshops. He received seven Prestigious Young Investigator Awards from NAE, ONR, ARO, IEEE, INNS, UIUC, Grainger Foundation; eleven Best Paper Awards from IEEE, ACM, IAPR, SPIE, SIAM; many major Industrial Research Awards from Google, Samsung, Amazon, Konica Minolta, JP Morgan, Zebra, Adobe, and Mathworks, etc. He is currently an Associate Editor of the IEEE Transactions on Neural Networks and Leaning Systems. He is fellow of IEEE, IAPR, OSA and SPIE, a Lifetime Distinguished Member of ACM, Lifetime Member of AAAI, and Institute of Mathematical Statistics, member of Global Young Academy, INNS and Beckman Graduate Fellow during 2007-2008. 